\title{
Towards Reconstructing the Quantum Effective Action of Gravity
}

\author{
Benjamin Knorr ${ }^{*}$ and Frank Saueressig ${ }^{\dagger}$ \\ Institute for Mathematics, Astrophysics and Particle Physics (IMAPP), Radboud University Nijmegen, \\ Heyendaalseweg 135, 6525 AJ Nijmegen, Netherlands
}

(Received 16 April 2018; revised manuscript received 6 July 2018; published 19 October 2018)

\begin{abstract}
Starting from a parametrization of the quantum effective action for gravity, we calculate correlation functions for observable quantities. The resulting templates allow us to reverse engineer the couplings describing the effective dynamics from the correlation functions. Applying this new formalism to the autocorrelation function of spatial volume fluctuations measured within the causal dynamical triangulations program suggests that the corresponding quantum effective action consists of the Einstein-Hilbert action supplemented by a nonlocal interaction term. We expect that our matching-template formalism can be adapted to a wide range of quantum gravity programs allowing us to bridge the gap between the fundamental formulation and observable low-energy physics.
\end{abstract}

DOI: 10.1103/PhysRevLett.121.161304

Introduction.-A characteristic feature of quantum gravity research is its fragmentation into disjoint branches including, e.g., string theory [1,2], loop quantum gravity $[3,4]$, the asymptotic safety program [5-8], causal dynamical triangulations (CDT) [9], causal set theory [10,11], group field theory $[12,13]$, and nonlocal gravity theories [14-16]. Each approach formulates its own fundamental dynamics typically at the Planck scale. The complexity of these formulations makes it quite hard to derive physical consequences from the fundamental formulation. (Any candidate for a theory of quantum gravity that reproduces general relativity in the infrared gives rise to universal oneloop corrections to, e.g., the Newton potential. However, it is highly nontrivial that a given microscopic prescription based, e.g., on a discretized spacetime structure or the violation of Lorentz symmetry at trans-Planckian scales, admits such a limit.)

A canonical way towards addressing this problem would be the construction of the quantum effective action $\Gamma$ which encodes the dynamics of a quantum theory taking all quantum fluctuations into account. In this way, it stores the outcome of a large number of (scattering) processes in an economical way. Generally, finding the exact form of $\Gamma$ is considered equivalent to solving the theory. Not surprisingly, calculating the quantum effective action is a hard problem. While fundamental Lagrangians describing our known physical theories are local and often restricted to a small number of interaction terms, $\Gamma$ generically contains

Published by the American Physical Society under the terms of the Creative Commons Attribution 4.0 International license. Further distribution of this work must maintain attribution to the author(s) and the published article's title, journal citation, and DOI. Funded by SCOAP ${ }^{3}$. all possible interactions permitted by the symmetries of the theory. Furthermore, quantum corrections related to massless particles like the graviton give rise to nonlocal terms [17].

In contrast, correlation functions built from fluctuations of physical quantities like volumes of (sub-) manifolds $\Sigma$, $V_{n}=\int_{\Sigma} d^{n} x \sqrt{g}$, and curvatures are accessible even at the nonperturbative level [18-21]. In this Letter, we explicitly demonstrate that this information allows us to reconstruct (parts of) the underlying quantum effective action, thereby taking a first explicit step in such a reconstruction program. Starting from the two-point autocorrelation functions of three-volume fluctuations measured in Monte Carlo simulations within CDT [18-21], we determine several couplings appearing in $\Gamma$. Our analysis provides first-hand evidence for the presence of nonlocal terms which could affect the gravitational dynamics at cosmic scales.

The quantum effective action for gravity.-In the case of gravity, the quantum effective action may be built from the spacetime metric and its curvature tensors. The local part of the quantum effective action can then be organized in terms of a derivative expansion. The lowest order terms coincide with the Einstein-Hilbert action

$$
\Gamma^{\text {local }}=\frac{1}{16 \pi G_{N}} \int d^{4} x \sqrt{g}[2 \Lambda-R]+\ldots
$$

where $G_{N}$ and $\Lambda$ are Newton's constant and the cosmological constant. The dots represent terms containing four or more derivatives as $\int d^{4} x \sqrt{g} R^{2}$, or the Goroff-Sagnotti counterterm [22-24] which will not be resolved here.

The nonlocal (NL) part of $\Gamma$ typically contains inverse powers of the Laplacian $\square \equiv-g^{\mu \nu} D_{\mu} D_{\nu}$ acting on curvature tensors. At second order in the curvature, this leads to 
terms of the form (For UV modifications of gravity including these types of form factors, see, e.g., $[25,26]$.)

$$
\Gamma^{\mathrm{NL}} \propto \int d^{4} x \sqrt{g} \mathcal{R} \mathcal{F}(\square) \mathcal{R},
$$

where $\mathcal{R}$ is linear in the curvature tensors and the functions $\mathcal{F}(\square)$ are known as form factors. (The form factors $\mathcal{F}(\square)$, defined through the matrix elements $\langle x|\mathcal{F}(\square)| y\rangle \equiv L(x-y)$ allow us to write nonlocal terms $\int d^{4} x \sqrt{g(x)} \int d^{4} y \sqrt{g(y)} R(x) L(x-y) R(y)$ into quasilocal form. Regularity of the nonlocal terms may require supplementing the operator appearing in the structure functions by nontrivial endomorphism terms built from the curvature [27]. We do not include them in the expressions (2), since their resolution depends on correlators which are beyond the scope of this Letter.) For the purpose of this Letter, we focus on diffeomorphism-invariant contributions which are quadratic in the curvature tensor and contain two inverse powers of the Laplacian, $\mathcal{F}(\square)=\square^{-2}$. This class contains the two independent terms

$$
\begin{aligned}
\Gamma_{R}^{\mathrm{NL}} & =-\frac{b^{2}}{96 \pi G_{N}} \int d^{4} x \sqrt{g} R \square^{-2} R, \\
\Gamma_{C}^{\mathrm{NL}} & =-\frac{\tilde{b}^{2}}{96 \pi G_{N}} \int d^{4} x \sqrt{g} C_{\mu \nu \rho \sigma} \square^{-2} C^{\mu \nu \rho \sigma} .
\end{aligned}
$$

Using the Bianchi identity satisfied by the Riemann tensor, $\int d^{4} x \sqrt{g} R^{\mu \nu} \square^{-2} R_{\mu \nu}$ can be rewritten in terms of these combinations and higher-order curvature terms and, thus, is not considered in the present construction. The characteristic feature of the nonlocal terms (3) is that they contribute mass terms to the propagators of scalar [Eq. (3a)] and graviton fluctuations [Eq. (3b)] when expanded around flat space. Thus, Eq. (3a) is the only diffeomorphisminvariant combination giving rise to a mass-type contribution to the two-point function in a toroidal background studied below. The task at hand is, then, to derive the values of the parameters $G_{N}, \Lambda, b, \tilde{b}, \ldots$, in terms of the parameters defining the fundamental theory. (For the implementation of this strategy in effective field theory, see [28].) In general, the latter set will vary from theory to theory. For CDT, they are given by the bare Newton's constant $\kappa_{0}$ and the relative size of spatial and timelike lines encoded in $\Delta$ [9].

Derivation of the matching template.-The foliation structure, constituting an elementary building block in the CDT program $[29,30]$, suggests to write $\Gamma$ using the Arnowitt-Deser-Misner formalism, reviewed, e.g., in [31]. In this case, the spacetime metric $g_{\mu \nu}$ is decomposed into a lapse function, a shift vector, and a metric $\sigma_{i j}$ measuring distances on spatial slices $\Sigma$ orthogonal to a normal vector $n_{\mu}$. Curvature tensors constructed from $g_{\mu \nu}$ can be separated into terms containing the intrinsic and extrinsic curvatures defined with respect to the foliation. In particular, the volume of the spatial slices,

$$
V_{3}(t) \equiv \int_{\Sigma} d^{3} x \sqrt{\sigma(t, x)}
$$

where $\sigma$ denotes the determinant of $\sigma_{i j}$, is invariant with respect to a change of coordinates on the spatial slice and constitutes an observable once the foliation is fixed.

CDT simulations have been performed for spatial slices $\bar{\Sigma}$ possessing the topology of a three sphere [18] and also, recently, for toroidal geometry [19-21]. Quite remarkably, the resulting profiles for the expectation value of three volumes $V_{3}(\bar{\Sigma}, t)$ as a function of the Euclidean time parameter $t \in[0,1)$ agrees with spacetime metrics of the form

$$
\bar{g}_{\mu \nu}=\operatorname{diag}\left[1, a(t)^{2} \bar{\sigma}_{i j}(x)\right],
$$

where

$$
\begin{array}{lll}
\text { torus: } & a(t)=1, & \bar{\sigma}_{i j}=\delta_{i j}, \\
\text { 3-sphere: } & a(t)=\sin (\pi t), & \bar{\sigma}_{i j}(x)=\bar{\sigma}_{i j}^{S^{3}}(x),
\end{array}
$$

with $\bar{\sigma}_{i j}^{S^{3}}(x)$ the standard metric of the three sphere. For concreteness, we will focus mainly on the toroidal case and only briefly comment on the analogous analysis for the spherical case. In the former case, the measured volume profile is essentially flat. Requiring that $\bar{\Sigma}=S^{1} \times S^{1} \times S^{1}$ is a solution to the equations of motion then fixes $\Lambda=0$. The higher-derivative and nonlocal terms in the quantum effective action do not contribute to the dynamics for this case.

Motivated by the existence of a well-defined background geometry, one can then study the autocorrelation of threevolume fluctuations around the background,

$$
\mathfrak{V}_{2}\left(t^{\prime}, t\right)=\left\langle\delta V_{3}\left(t^{\prime}\right) \delta V_{3}(t)\right\rangle
$$

Based on the quantum effective action, the fluctuations in the spatial metric are defined in the standard way, setting

$$
\sigma_{i j}(t, x)=\bar{\sigma}_{i j}+\delta \sigma_{i j}(t, x) .
$$

The fluctuations in the three volume can then be found by expanding $V_{3}(\Sigma, t) \equiv \int d^{3} x \sqrt{\sigma}$ in powers of the fluctuations. To leading order,

$$
\delta V_{3}(t)=\frac{1}{2} \int d^{3} x \sqrt{\bar{\sigma}} \bar{\sigma}^{i j} \delta \sigma_{i j}+\mathcal{O}\left(\delta \sigma^{2}\right)
$$

Introducing the fluctuation field $\hat{\sigma}(t, x) \equiv \bar{\sigma}^{i j} \delta \sigma_{i j}(t, x)$, the correlator $\mathfrak{V}_{2}$ is given by the integral over the propagator $\mathfrak{G}_{\hat{\sigma} \hat{\sigma}}\left(t^{\prime}, x^{\prime} ; t, x\right) \equiv\left\langle\hat{\sigma}\left(t^{\prime}, x^{\prime}\right) \hat{\sigma}(t, x)\right\rangle$ 
$\mathfrak{V}_{2}\left(t^{\prime}, t\right)=\frac{1}{4} \int d^{3} x^{\prime} \sqrt{\bar{\sigma}} \int d^{3} x \sqrt{\bar{\sigma}}\left\langle\hat{\sigma}\left(t^{\prime}, x^{\prime}\right) \hat{\sigma}(t, x)\right\rangle$.

The computation of the two-point function proceeds by expanding the quantum effective action to second order in $\hat{\sigma}, \Gamma^{\text {quad }}=(1 / 32 \pi G) \int d^{4} x \sqrt{\bar{g}} \hat{\sigma} \Gamma^{(2)} \hat{\sigma}$. The propagator $\mathfrak{G}_{\hat{\sigma} \hat{\sigma}}\left(t^{\prime}, x^{\prime} ; t, x\right)$ can then be expressed in terms of the eigenvalue spectrum $\left\{\bar{\lambda}_{n}\right\}$ and normalized eigenfunctions $\Phi_{n}(t, x)$ of the differential operator $\Gamma^{(2)}$,

$$
\mathfrak{G}=16 \pi G_{N} \sum_{n} \frac{1}{\bar{\lambda}_{n}} \Phi_{n}^{*}\left(t^{\prime}, x^{\prime}\right) \Phi_{n}(t, x) .
$$

For compact spaces and correlation functions involving fluctuations which are averaged over $\bar{\Sigma}$, the construction of the two-point function can be simplified by the following observation. On compact spaces, the eigenfunctions $\Phi_{n}(t, x)$ can be expanded in a complete set of orthonormal functions $\psi_{k}(x)$ defined on $\bar{\Sigma}$,

$$
\Phi_{n}(x, t)=\sum_{k} \phi_{n, k}(t) \psi_{k}(x) .
$$

The spatial integrals appearing in (10) then project the expansion (12) on the spatially constant mode $\psi_{0}(x) \equiv\left[V_{3}(\bar{\Sigma})\right]^{-1 / 2}$. Hence,

$$
\mathfrak{B}_{2}\left(t^{\prime}, t\right)=4 \pi G_{N} V_{3}(\bar{\Sigma}) \sum_{n}^{\prime} \frac{1}{\lambda_{n}} \phi_{n}^{*}\left(t^{\prime}\right) \phi_{n}(t),
$$

where $\left\{\lambda_{n}\right\}$ is the eigenvalue spectrum of $\Gamma^{(2)}$ restricted to constant spatial modes. The prime indicates that the zero mode should be excluded since it corresponds to an overall rescaling of the volume.

The next step computes $\Gamma^{(2)}$ by expanding the local and nonlocal terms in $\Gamma$ given by (1) and (2) to second order in $\hat{\sigma}$. Restricting to fluctuations which are constant on $\bar{\Sigma}$, the result reads

$$
\Gamma^{(2)}=\frac{1}{3}\left[\partial_{t}^{2}-b^{2}+\frac{1}{2} \Lambda\right] .
$$

Notably, this expression does not contain $\tilde{b}$, indicating that the correlator (10) does not carry information about the nonlocal graviton mass term.

Constructing $\mathfrak{V}_{2}\left(t^{\prime}, t\right)$ on a toroidal background requires the eigenvalues and eigenfunctions of $\Gamma^{(2)}$, solving (The mode $\hat{\sigma}$ comes with a wrong-sign kinetic term. Following the CDT study [18], we consider the negative of the corresponding operator in the sequel.)

$$
-\phi_{n}^{\prime \prime}(t)+b^{2} \phi_{n}(t)=\lambda_{n} \phi_{n}(t) .
$$

The solution is readily given in terms of Fourier modes $\phi_{n}(t)=e^{2 \pi \mathbf{i} n t}, \quad \lambda_{n}=(2 \pi n)^{2}+b^{2}, \quad n \in \mathbb{Z}$.

Based on the spectrum (16), the propagator can be readily calculated. Carrying out the sum gives

$$
\begin{aligned}
\sum_{n}^{\prime} & \frac{1}{\lambda_{n}} \phi_{n}^{*}\left(t^{\prime}\right) \phi_{n}(t) \\
= & \frac{e^{2 \pi \mathbf{i}\left(t^{\prime}-t\right)}}{2 b\left(4 \pi^{2}+b^{2}\right)}\left[( b - 2 \pi \mathbf { i } ) _ { 2 } F _ { 1 } \left(1,1-\frac{\mathbf{i} b}{2 \pi} ; 2\right.\right. \\
& \left.\left.-\frac{\mathbf{i} b}{2 \pi} \mid e^{2 \pi \mathbf{i}\left(t^{\prime}-t\right)}\right)-(b \rightarrow-b)\right]+ \text { c.c. }
\end{aligned}
$$

For $b^{2}<4 \pi^{2}$, the sum can be expanded in a convergent power series in $b^{2}$ whose coefficients contain polylogarithms:

$$
\begin{aligned}
\sum_{n}^{\prime} & \frac{1}{\lambda_{n}} \phi_{n}^{*}\left(t^{\prime}\right) \phi_{n}(t) \\
\quad= & \sum_{n=1}^{\infty}\left[\operatorname{Li}_{2 n}\left(e^{2 \pi \mathbf{i}\left(t^{\prime}-t\right)}\right)+\operatorname{Li}_{2 n}\left(e^{-2 \pi \mathbf{i}\left(t^{\prime}-t\right)}\right)\right]\left(\frac{b^{2}}{4 \pi^{2}}\right)^{n-1} .
\end{aligned}
$$

Thus, the limit $b \rightarrow 0$ is continuous and finite. The expansion for small time steps $\left|t^{\prime}-t\right| \ll 1$ yields

$\sum_{n}^{\prime} \frac{1}{\lambda_{n}} \phi_{n}^{*}\left(t^{\prime}\right) \phi_{n}(t) \approx \frac{b \operatorname{coth}(b / 2)-2}{2 b^{2}}-\frac{\left|t^{\prime}-t\right|}{2}+\cdots$,

so that the height of the peak is determined by $b$.

Comparison with CDT data.-Now, we are in the situation that we can compare to the data obtained from CDT simulations on the torus for bare parameters [cf. the discussion below Eq. (3)]

$$
\kappa_{0}=2.2, \quad \Delta=0.6,
$$

and configurations built from $N_{4}=160000$ simplices [19]. This point is located well within the de Sitter phase of the CDT phase diagram [21]. Averaging over all times, we can fit the correlator $\mathfrak{V}_{2}(t, t+\Delta t)$ to extract the value of Newton's constant $G_{N}$ and the mass parameter $b^{2}$. A least squares fit gives

$G_{N}=0.14 a_{\mathrm{CDT}}^{2}, \quad \ell_{\mathrm{PL}}=0.37 a_{\mathrm{CDT}}, \quad b=6.93 / a_{\mathrm{CDT}}$.

Here, $a_{\mathrm{CDT}}$ is the lattice spacing and $\ell_{\mathrm{PL}} \equiv \sqrt{G_{N}}$ is the Planck length (PL). We display the lattice data (blue dots) and our fit (red line) in Fig. 1, and find a very good agreement between the two.

The relation between the lattice spacing and the physical radius $r$ of the torus can be obtained by fitting the 


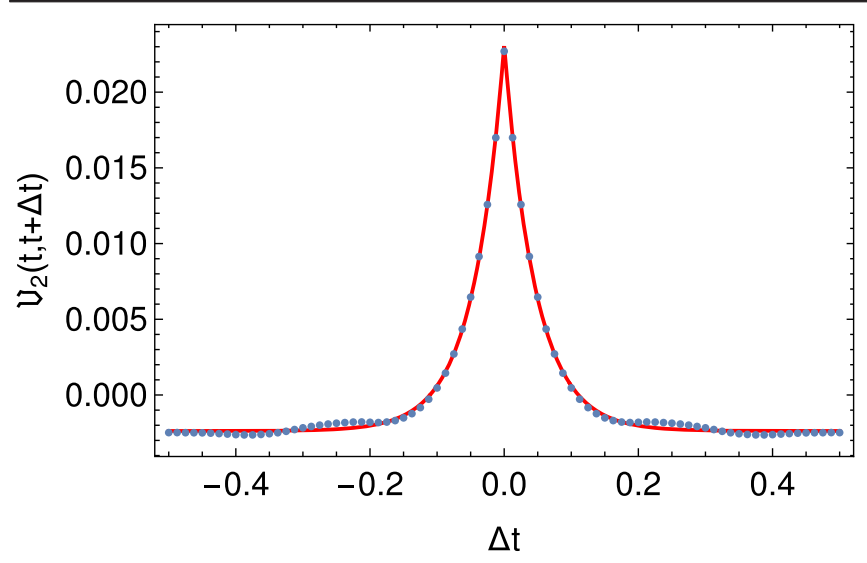

FIG. 1. The correlator $\mathfrak{V}_{2}(t, t+\Delta t)$ on the torus in arbitrary units. Blue dots are the lattice data (averaged over all temporal slices), the red line indicates the fitted analytical result.

eigenvalues of the covariance matrix to the analytic form (16). Since the higher eigenvalues are less precise, we only fit the lowest three eigenvalues. Demanding that the resulting Newton's constant agrees with the one from the fit, gives a relation between the physical radius and the lattice spacing

$$
r=3.09 a_{\mathrm{CDT}} .
$$

The value of $b$ can also be calculated from the lattice data directly by resorting to an action for the volume fluctuations inspired by a minisuperspace computation

$$
b_{\mathrm{CDT}}=\sqrt{\frac{\bar{\Gamma} u}{\gamma(1+\gamma)}} \bar{V}_{3} .
$$

Here, $\bar{\Gamma}$ is related to the kinetic term, $u$ to the potential term, and $\gamma$ is a critical exponent. For the data point (20), we take $\bar{\Gamma}=26.3, u=1.30 \times 10^{-6}$ ("first average then invert"), $\bar{V}_{3}=2000$, and $\gamma=1.16$ given in [19], and find

$$
b_{\mathrm{CDT}}=(7.39 \pm 0.84 \pm 0.14 \Delta \bar{\Gamma}) / a_{\mathrm{CDT}},
$$

which is consistent with the fit value within numerical precision. No numerical error $\Delta \bar{\Gamma}$ has been given in [19], and we leave it unspecified here.

Naturally, one would expect that the local part of the quantum effective action also contains higher-derivative terms like $\int d^{4} x \sqrt{g} R^{2}$ containing four (or more) spacetime derivatives. On the toroidal background, the Hessian $\Gamma^{(2)}$ then acquires an additional term proportional to $\partial_{t}^{4}$. Including this contribution in the fitting procedure shows that the related coefficient is negligible though.

Adapting the construction (13) to backgrounds where $\bar{\Sigma}=S^{3}$ leads to the eigenproblem studied in [18]. The authors have shown there that the resulting fluctuation spectrum agrees very well with the numerical data. For the configuration (20) a comparison of the lowest eigenvalue of the covariance matrix with the continuum version yields

$$
G_{N}=0.23 a_{\mathrm{CDT}}^{2}, \quad \ell_{\mathrm{PL}}=0.48 a_{\mathrm{CDT}} .
$$

The relation between the lattice spacing and the physical radius $r=3.1 a_{\mathrm{CDT}}$ is taken from [18] and agrees with the relation on the torus at the same bare parameters. Thus, our continuum approach also reproduces the Monte Carlo results obtained for spherical topology.

Background independence of the quantum effective action suggests that the values for $G_{N}$ obtained in different simulations should agree. The comparison of the Newton's constant found for the toroidal (21) and spherical topology (25) indicates different values though. This puzzle is resolved by the observation that the couplings $G_{N}$ obtained from the volume correlations on the torus and spherical background are actually not the same: for nonvanishing background curvature, higher-order curvature terms contribute to the $\partial_{t}^{2}$ terms appearing in $\Gamma^{(2)}$, so that the $G_{N}$ obtained in (25) is actually a function of the Newton's constant defined in (1) and higher-derivative couplings. Likewise, the fact that the data obtained for $\bar{\Sigma}=S^{3}$ suggest $b=0$ is not in contradiction with the toroidal results since the correlator of volume fluctuations evaluated on a background with nonzero curvature is not sensitive to this coupling. Instead, it probes nontrivial endomorphism terms regulating the inverse Laplacians on a constant curvature background [27], which come with their own couplings.

Information from complementary correlators.-The two-point autocorrelation function (7) gives access to some couplings appearing in the quantum effective action. A more complete picture can be developed by either studying correlation functions of different geometrical quantities or higher-order $n$-point functions.

Higher-order $n$-point functions: A natural generalization of (7) is higher-order correlators involving the fluctuations of spatial volumes at $n$ time steps

$$
\mathfrak{B}_{n}\left(t_{1}, \ldots, t_{n}\right) \equiv\left\langle\delta V_{3}\left(t_{1}\right) \ldots \delta V_{3}\left(t_{n}\right)\right\rangle
$$

These correlators can be constructed systematically by taking derivatives of $\mathfrak{V}_{2}$ with respect to a suitable source. The three-point correlator $\mathfrak{V}_{3}$, for instance, then involves the three-point vertex contracted with three propagators. On a flat, toroidal background, $\mathfrak{V}_{n}\left(t_{1}, \ldots, t_{n}\right)$ carries information on couplings associated with terms built from $n$ powers of the Riemann tensor (and its contractions).

Two-point functions involving curvatures: Complementarily, one may study the autocorrelation of curvature fluctuations involving the extrinsic or intrinsic curvature. Focusing on one concrete example, we introduce the averaged intrinsic curvature 


$$
\mathcal{R}_{3}(t)=\int d^{3} x \sqrt{\sigma}^{(3)} R
$$

The analogue of (9) is then obtained by expanding $\mathcal{R}_{3}(t)$ in terms of fluctuations

$$
\begin{aligned}
\tilde{\sigma}(x, t) & =a(t) \bar{\sigma}^{\mu \nu}(x) \delta \sigma_{\mu \nu}(x, t), \\
\tilde{\sigma}_{\mu \nu}^{\mathrm{TL}}(x, t) & =a(t) \delta \sigma_{\mu \nu}(x, t)-\frac{1}{3} \bar{\sigma}_{\mu \nu}(x) \tilde{\sigma}(x, t) .
\end{aligned}
$$

The superscript TL indicates that the given tensor is traceless. Dropping integrals over total derivatives, fluctuations of the averaged intrinsic curvature are related to the fluctuation fields by

$$
\delta \mathcal{R}_{3}(t)=\int d^{3} x \sqrt{\bar{\sigma}}\left(\frac{1}{6}{ }^{(3)} \tilde{R} \tilde{\sigma}-{ }^{(3)} \tilde{S}^{\mu \nu} \tilde{\sigma}_{\mu \nu}^{\mathrm{TL}}\right),
$$

where ${ }^{(3)} \bar{R}={ }^{(3)} \tilde{R} / a(t)^{2}$ and ${ }^{(3)} \bar{S}^{\mu \nu}={ }^{(3)} \tilde{S}^{\mu \nu} / a(t)^{4}$ indicate the background spatial Ricci scalar and trace-free spatial Ricci tensor, respectively. The autocorrelation function can then again be expressed in terms of the propagators of the fluctuation fields,

$$
\begin{aligned}
& \left\langle\delta \mathcal{R}_{3}\left(t^{\prime}\right) \delta \mathcal{R}_{3}(t)\right\rangle \\
& =\int d^{3} x \sqrt{\bar{\sigma}} \int d^{3} y \sqrt{\bar{\sigma}}\left(\frac{{ }^{(3)} \tilde{R}^{2}}{36} \mathfrak{G}_{\tilde{\sigma} \tilde{\sigma}}-\frac{1}{3}{ }^{(3)} \tilde{R}^{(3)} \tilde{S}^{\mu \nu}\left(\mathfrak{G}_{\tilde{\sigma} \tilde{\sigma}^{\mathrm{TL}}}\right)_{\mu \nu}\right. \\
& \left.\quad+{ }^{(3)} \tilde{S}^{\mu \nu(3)} \tilde{S}^{\rho \sigma}\left(\mathfrak{G}_{\tilde{\sigma}^{\mathrm{TL}} \tilde{\sigma}^{\mathrm{TL}}}\right)_{\mu \nu \rho \sigma}\right)
\end{aligned}
$$

Notably, $\left\langle\delta \mathcal{R}_{3}\left(t^{\prime}\right) \delta \mathcal{R}_{3}(t)\right\rangle$ vanishes on a toroidal background since it is proportional to the background curvature. This feature is owed to terminating the expansion (29) at leading order in the fluctuation fields. Once terms quadratic in the fluctuations are included, the correlator (30) involves nonzero contributions related to the four-point vertex of the fluctuation fields.

Conclusions. - We introduced a new research program to reverse-engineer the quantum effective action for gravity from correlation functions. This provides, for the first time, a direct link between continuum and lattice in quantum gravity beyond abstract quantities like critical exponents [32,33] and spectral dimensions [34-37]. Where lattice data were available, agreement with an Einstein-Hilbert action, potentially amended by a particular nonlocal interaction or higher-order scalar curvature terms, was found.

A particularly intriguing result is that the lattice simulations on the torus suggest the presence of a (nonlocal) mass term. The authors of [19] argue that the occurrence of this term is a genuine quantum gravity effect. Generically, it is expected that quantum fluctuations of massless particles induce these kind of nonlocal terms in the quantum effective action. A prototypical example is provided by quantum chromodynamics where such terms correctly describe the nonperturbative gluon propagator in the IR [38-41].

In general, it is conceivable that nonlocal gravitational interactions provide a dynamical explanation of dark energy, without the need for a fine-tuned cosmological constant [42-44]. In particular, the nonlocal contribution given in Eq. (3) forms a key part of the MaggioreMancarella cosmological model [43,45], which has been highly successful in describing the cosmological evolution of the Universe. (For earlier Letter discussing the effect of nonlocal terms in cosmology, see, also, [46].) It is intriguing that the nonclassical behavior seen on the lattice is compatible with such a nonlocal quantum effect. Since Eq. (4) is known to lead to unstable modes in the cosmic perturbation spectrum [47], it would be highly interesting to determine $\tilde{b}$ from the fundamental formulation. This is beyond the present Letter though.

In this Letter, we analyzed the arguably simplest nontrivial correlation function describing the autocorrelation of three-volume fluctuations at two different times (7). The systematic extension to correlation functions of higher order or other structures is evident. Notably, a measurement of the correlators (26) and (30) may actually be feasible within the CDT program, thereby providing further information on the quantum effective action. In particular, correlation functions of the averaged intrinsic curvature may be obtained by summing deficit angles or new sophisticated methods to measure curvature on general quantum spacetimes recently introduced in $[48,49]$. We stress that the construction of reverse engineering the quantum effective action from correlators is not limited to CDT, but applies to any given theory of quantum gravity in which the corresponding quantities can be computed.

We thank J. Ambjørn, A. Görlich, and R. Loll for interesting discussions, and A. Görlich for providing us with the numerical data from the CDT simulations. Interesting discussions with J. Donoghue, N. Klitgaard, and M. Maggiore are acknowledged. This research is supported by the Netherlands Organisation for Scientific Research (NWO) within the Foundation for Fundamental Research on Matter (FOM) Grant No. 13VP12.

*b.knorr@science.ru.nl

f.saueressig@science.ru.nl

[1] K. Becker, M. Becker, and J. H. Schwarz, String Theory and M-Theory: A Modern Introduction (Cambridge University Press, Cambridge, England, 2006).

[2] V. Schomerus, A Primer on String Theory (Cambridge University Press, Cambridge, England, 2017).

[3] T. Thiemann, Lectures on loop quantum gravity, in Proceedings, 271st WE-Heraeus Seminar on Aspects of Quantum Gravity: From Theory to Experiment Search: Bad Honnef, Germany, 2002, Lecture Notes in Physics Vol. 631 (Springer, Berlin, 2003), pp. 41-135. 
[4] C. Rovelli, Quantum Gravity (Cambridge University Press, Cambridge, England, 2004).

[5] M. Niedermaier and M. Reuter, The asymptotic safety scenario in quantum gravity, Living Rev. Relativity 9, 5 (2006).

[6] M. Reuter and F. Saueressig, Quantum Einstein gravity, New J. Phys. 14, 055022 (2012).

[7] R. Percacci, An Introduction to Covariant Quantum Gravity and Asymptotic Safety, 100 Years of General Relativity, Vol. 3 (World Scientific, Singapore, 2017).

[8] A. Eichhorn, Status of the asymptotic safety paradigm for quantum gravity and matter: Black holes, gravitational waves and spacetime singularities, Found. Phys. 48, 1407 (2018).

[9] J. Ambjorn, A. Goerlich, J. Jurkiewicz, and R. Loll, Nonperturbative Quantum Gravity, Phys. Rep. 519, 127 (2012).

[10] R. D. Sorkin, Causal sets: Discrete gravity, in Lectures on quantum gravity. Proceedings, School of Quantum Gravity, Valdivia, Chile, 2002, edited by A. Gomberoff and D. Marolf (Springer, Boston, 2005), pp. 305-327.

[11] S. Surya, Directions in Causal Set Quantum Gravity (to be published).

[12] A. Baratin and D. Oriti, Group Field Theory with Noncommutative Metric Variables, Phys. Rev. Lett. 105, 221302 (2010).

[13] D. Oriti, The microscopic dynamics of quantum space as a group field theory, in Proceedings, Foundations of Space and Time: Reflections on Quantum Gravity: Cape Town, South Africa 2011 (Cambridge University Press, Cambridge, 2012), ISBN: 9780521114400, pp. 257-320.

[14] T. Biswas, A. Mazumdar, and W. Siegel, Bouncing universes in string-inspired gravity, J. Cosmol. Astropart. Phys. 03 (2006) 009.

[15] L. Modesto, Superrenormalizable quantum gravity, Phys. Rev. D 86, 044005 (2012).

[16] L. Modesto and L. Rachwał, Nonlocal quantum gravity: A review, Int. J. Mod. Phys. D 26, 1730020 (2017).

[17] J. F. Donoghue, M. M. Ivanov, and A. Shkerin, EPFL Lectures on General Relativity as a Quantum Field Theory, arXiv: 1702.00319.

[18] J. Ambjorn, A. Gorlich, J. Jurkiewicz, and R. Loll, The nonperturbative quantum de Sitter universe, Phys. Rev. D 78, 063544 (2008).

[19] J. Ambjorn, Z. Drogosz, J. Gizbert-Studnicki, A. Görlich, J. Jurkiewicz, and D. Nemeth, Impact of topology in causal dynamical triangulations quantum gravity, Phys. Rev. D 94, 044010 (2016).

[20] J. Ambjørn, J. Gizbert-Studnicki, A. Görlich, K. Grosvenor, and J. Jurkiewicz, Four-dimensional CDT with toroidal topology, Nucl. Phys. B922, 226 (2017).

[21] J. Ambjørn, J. Gizbert-Studnicki, A. Görlich, J. Jurkiewicz, and D. Németh, The phase structure of causal dynamical triangulations with toroidal spatial topology, J. High Energy Phys. 06 (2018) 111.

[22] M. H. Goroff and A. Sagnotti, Quantum gravity at two loops, Phys. Lett. 160B, 81 (1985).

[23] M. H. Goroff and A. Sagnotti, The ultraviolet behavior of Einstein gravity, Nucl. Phys. B266, 709 (1986).

[24] H. Gies, B. Knorr, S. Lippoldt, and F. Saueressig, Gravitational Two-Loop Counterterm Is Asymptotically Safe, Phys. Rev. Lett. 116, 211302 (2016).
[25] T. Biswas, E. Gerwick, T. Koivisto, and A. Mazumdar, Towards Singularity and Ghost Free Theories of Gravity, Phys. Rev. Lett. 108, 031101 (2012).

[26] J. Edholm, A. S. Koshelev, and A. Mazumdar, Behavior of the Newtonian potential for ghost-free gravity and singularity-free gravity, Phys. Rev. D 94, 104033 (2016).

[27] A. O. Barvinsky, Nonlocal gravity, Schwinger-Keldysh technique, dark energy and all that, in Proceedings, 17th International Seminar on High Energy Physics (Quarks 2012): Yaroslavl, Russia, 2012 (Institute for Nuclear Research, Moscow, Russia, 2012).

[28] J. F. Donoghue and B. K. El-Menoufi, Covariant non-local action for massless QED and the curvature expansion, J. High Energy Phys. 10 (2015) 044.

[29] J. Ambjorn and R. Loll, Nonperturbative Lorentzian quantum gravity, causality and topology change, Nucl. Phys. B536, 407 (1998).

[30] J. Ambjorn, J. Jurkiewicz, and R. Loll, Emergence of a 4-D World from Causal Quantum Gravity, Phys. Rev. Lett. 93, 131301 (2004).

[31] E. Gourgoulhon, $3+1$ formalism and bases of numerical relativity, arXiv:gr-qc/0703035.

[32] K. Falls, Critical scaling in quantum gravity from the renormalisation group, arXiv:1503.06233.

[33] J. Biemans, A. Platania, and F. Saueressig, Quantum gravity on foliated spacetimes: Asymptotically safe and sound, Phys. Rev. D 95, 086013 (2017).

[34] J. Ambjorn, J. Jurkiewicz, and R. Loll, Spectral Dimension of the Universe, Phys. Rev. Lett. 95, 171301 (2005).

[35] O. Lauscher and M. Reuter, Fractal spacetime structure in asymptotically safe gravity, J. High Energy Phys. 10 (2005) 050.

[36] D. Benedetti and J. Henson, Spectral geometry as a probe of quantum spacetime, Phys. Rev. D 80, 124036 (2009).

[37] G. Clemente and M. D'Elia, Spectrum of the LaplaceBeltrami operator and the phase structure of causal dynamical triangulations, Phys. Rev. D 97, 124022 (2018).

[38] P. Boucaud, A. Le Yaouanc, J. P. Leroy, J. Micheli, O. Pene, and J. Rodriguez-Quintero, Testing Landau gauge OPE on the lattice with a $<A^{2}>$ condensate, Phys. Rev. D 63, 114003 (2001).

[39] M. A. L. Capri, D. Dudal, J. A. Gracey, V. E. R. Lemes, R. F. Sobreiro, S. P. Sorella, and H. Verschelde, A Study of the gauge invariant, nonlocal mass operator $\mathrm{Tr}$ $\int d^{4} x F_{\mu \nu}\left(D^{2}\right)^{-1} F_{\mu \nu}$ in Yang-Mills theories, Phys. Rev. D 72, 105016 (2005).

[40] D. Dudal, J. A. Gracey, S. P. Sorella, N. Vandersickel, and H. Verschelde, A Refinement of the Gribov-Zwanziger approach in the Landau gauge: Infrared propagators in harmony with the lattice results, Phys. Rev. D 78, 065047 (2008).

[41] M. Pelaez, M. Tissier, and N. Wschebor, Two-point correlation functions of QCD in the Landau gauge, Phys. Rev. D 90, 065031 (2014).

[42] M. Maggiore, Phantom dark energy from nonlocal infrared modifications of general relativity, Phys. Rev. D 89, 043008 (2014).

[43] M. Maggiore and M. Mancarella, Nonlocal gravity and dark energy, Phys. Rev. D 90, 023005 (2014). 
[44] S. Foffa, M. Maggiore, and E. Mitsou, Cosmological dynamics and dark energy from nonlocal infrared modifications of gravity, Int. J. Mod. Phys. A 29, 1450116 (2014).

[45] E. Belgacem, Y. Dirian, S. Foffa, and M. Maggiore, Nonlocal gravity. Conceptual aspects and cosmological predictions, J. Cosmol. Astropart. Phys. 03 (2018) 002.

[46] S. Deser and R. P. Woodard, Nonlocal Cosmology, Phys. Rev. Lett. 99, 111301 (2007).
[47] G. Cusin, S. Foffa, M. Maggiore, and M. Mancarella, Nonlocal gravity with a Weyl-square term, Phys. Rev. D 93, 043006 (2016).

[48] N. Klitgaard and R. Loll, Introducing quantum Ricci curvature, Phys. Rev. D 97, 046008 (2018).

[49] N. Klitgaard and R. Loll, Implementing quantum Ricci curvature, Phys. Rev. D 97, 106017 (2018). 